\title{
Design of a zinc finger protein binding a sequence upstream of the
}

\section{A20 gene}

\author{
Yong Wei ${ }^{1}$, Dajun Ying*1, Chunli Hou ${ }^{1}$, Xiaoping Cui ${ }^{1,2}$ and Chuhong Zhu*1
}

Address: ${ }^{1}$ The Key Laboratory of Biomechanics and Tissue Engineering of Chongqing Municipality, Department of Anatomy, Third Military Medical University, Chongqing, 400038, China and 2Department of Neurology, Fuzhou General Hospital, Fuzhou, 350025, China

Email: Yong Wei - yongwei70@gmail.com; Dajun Ying* - ydj96@yahoo.com.cn; Chunli Hou - houchunliwf@163.com;

Xiaoping Cui - xpcuitmmu@yahoo.com; Chuhong Zhu* - zhuch99@yahoo.com

* Corresponding authors

Published: 19 March 2008

BMC Biotechnology 2008, 8:28 doi:10.1/86/1472-6750-8-28
Received: 23 November 2007

Accepted: 19 March 2008

This article is available from: http://www.biomedcentral.com/I472-6750/8/28

(C) 2008 Wei et al; licensee BioMed Central Ltd.

This is an Open Access article distributed under the terms of the Creative Commons Attribution License (http://creativecommons.org/licenses/by/2.0), which permits unrestricted use, distribution, and reproduction in any medium, provided the original work is properly cited.

\begin{abstract}
Background: Artificial transcription factors (ATFs) are composed of DNA-binding and functional domains. These domains can be fused together to create proteins that can bind a chosen DNA sequence. To construct a valid ATF, it is necessary to design suitable DNA-binding and functional domains. The $\mathrm{Cys}_{2}-\mathrm{His}_{2}$ zinc finger motif is the ideal structural scaffold on which to construct a sequence-specific protein. A20 is a cytoplasmic zinc finger protein that inhibits nuclear factor kappa-B activity and tumor necrosis factor (TNF)-mediated programmed cell death. A20 has been shown to prevent TNF-induced cytotoxicity in a variety of cell types including fibroblasts, B lymphocytes, WEHI 164 cells, NIH 3T3 cells and endothelial cells.
\end{abstract}

Results: In order to design a zinc finger protein (ZFP) structural domain that binds specific target sequences in the $\mathrm{A} 20$ gene promoter region, the structure and sequence composition of this promoter were analyzed by bioinformatics methods. The target sequences in the A20 promoter were submitted to the on-line ZF Tools server of the Barbas Laboratory, Scripps Research Institute (TSRI), to obtain a specific 18 bp target sequence and also the amino acid sequence of a ZFP that would bind to it. Sequence characterization and structural modeling of the predicted ZFP were performed by bioinformatics methods. The optimized DNA sequence of this artificial ZFP was recombined into the eukaryotic expression vector pIRES2-EGFP to construct PIRES2-EGFP/ZFPflag recombinants, and the expression and biological activity of the ZFP were analyzed by RT-PCR, western blotting and EMSA, respectively. The ZFP was designed successfully and exhibited biological activity.

Conclusion: It is feasible to design specific zinc finger proteins by bioinformatics methods.

\section{Background}

In nature, gene expression is regulated at the transcriptional level primarily by transcription factors that bind to DNA. Many of these transcription factors consist of two essential yet separable modules: a DNA-binding domain and a functional domain [1-3]. Artificial transcription fac- tors (ATFs) are composed of DNA-binding and functional domains [4-6], which can be fused together to create proteins that bind a chosen DNA sequence and regulate expression of a specific gene in vivo $[1,2,4,7,8]$. Construction of ATFs in vitro includes construction of DNA-binding and function domains by various methods. It is very 
important to design a DNA-binding domain that recognizes a specific DNA sequence. Recently there has been a great deal of progress in the development of modular protein domains that recognize specific DNA triplets. The $\mathrm{Cys}_{2}$-His 2 zinc finger motif is the ideal structural scaffold on which a sequence-specific protein may be constructed $[7,9]$. DNA structural domains of zinc finger proteins (ZFPs) usually consist of 3 or 6 zinc fingers. Artificial ZFP (AZP) technology allows DNA sequences to be selected directionally and a DNA-binding domain to be designed [10-13].

A20 (Tumor Necrosis Factor Alpha-Induced Protein 3, TNFAIP3) is a cytoplasmic zinc finger protein that inhibits nuclear factor kappa-B (NFkB) activity and tumor necrosis factor (TNF)-mediated programmed cell death [14-16]. A20 has been shown to prevent TNF-induced cytotoxicity in a variety of cell types including fibroblasts, B-lymphocytes, WEHI 164 cells, NIH 3T3 cells and endothelial cells [17]. Over-expression of A20 inhibits IL-1 $\beta$-induced production of NO by rat islets, which impacts positively on islet graft survival and function [18]. Indeed, A20-deficient cells fail to terminate TNF-induced NF- $\kappa \mathrm{B}$ activation [19]. Lee et al. generated A20-deficient mice by targeted disruption. A20 -/- mice, born from interbred A20 +/mice in Mendelian ratios, developed runting as early as 1 week of age [13]. Mice deficient in A20 developed severe inflammation and cachexia, were hypersensitive to both lipopolysaccharide and TNF, and died prematurely.

In the present study, to provide a basis for designing ATFs that can regulate the human A20 gene, a ZFP sequence involving six contiguous $\mathrm{Cys}_{2} \mathrm{His}_{2}$-type zinc fingers was constructed using the classical $\mathrm{Cys}_{2} \mathrm{His}_{2}$-type zinc finger model and an 18 bp DNA sequence (as target sequence) obtained from the A20 gene promoter region.

\section{Results}

\section{Transcription start site and presumptive promoter sequence of $A 20$ gene}

The DNA sequence of the human A20 gene (-1000 bp +300 bp) was used to analyze the promoter region. Transacting factors and related elements that may interact with the aforementioned sequence were analyzed by the online TFSEARCH[20], TESS[21] and Gene2Promoter servers[22]. The results indicated many trans-acting factors binding the relevant elements in the gene sequence, with several HSF binding sites at the distal end and 6 SP1 and $2 \mathrm{NF}-\kappa \mathrm{B}$ binding sites in the GC-rich region at the proximal end.

The 1300 bp sequence was submitted to Gene2Promoter of Genomatix[22] to locate the A20 gene TSS. The results indicated 12 sequence fragments that may contain TSS sites. The features of these sequence fragments were com- pared with nucleic acid sequences of different histological origins, and the score of a 121 bp sequence within NC $000006138229984-138230104$ of the genome proved significantly higher than that of the other sequence fragments. Hence, this 121 bp sequence probably includes the TSS of the A20 gene. Further analysis of the 121 bp sequence showed that the score of the site in genomic NC 000006138230088 (the site in the frame...agactgcgcagtctg..., see Seq.1) reached a peak (Fig. 1A). Therefore, the TSS of the A20 gene can be determined by bioinformatics methods. The results of prediction were consistent with the conclusions that Krikos drew from an S1 Nuclease Protection Assay [23].

After the TSS of the A20 gene was determined, the established promoter sequence of this gene was submitted to Gene2Promoter of the Genomatix[22] and McPromoter[24] online server (Nuremberg University) to analyze the presumptive promoter sequence further. The results suggested that the presumptive promoter might be located within genomic NC 000006 138229781138230472 and be 692 bp in length. Moreover, analysis by the McPromoter program based on a Markov model suggested that the presumptive promoter may be a sequence approximately 300 bp in length, which is covered by the sequence predicted by Gene2Promoter (Fig. 1B).

These analyses defined the presumptive promoter sequence and TSS of the A20 gene. In the light of the following study design, a 396 bp sequence fragment within NC $000006138229786-138230182$ of the A20 gene promoter was selected as the target sequence for study (see Seq.1).

cttccgaaatgccaggtgactcacgcggggacacccogggcggggcgagggag tttctccgggcgcctgcagggaccgggcggggcggggcagcggggcggggcagggaaagggggcggggcggggccogcaggccoggtcgggcggaggccgcgcgcgcccctcgcccctgcgccctctggcggccggctggacgcacttcgca gccoaccagagagtcacgtgactttggaaagtcccgtggaaatcccoggcctacaacccgcatacaactgaaacggggcaaagcagactgcgcagtctgcagtcttcgtggcgggccaagcgagcttggagccogcgggggcggagcggtgagagcggc cgccaagagagatcacaccccagcc (396 bp)

\section{Procurement of target sequence of A20 gene promoter region and ZFP design}

The presumptive promoter sequence obtained for the A20 gene was submitted to the ZF tools server [25] to "Search DNA Sequence for Contiguous Target Sites". After setting parameters and comparing with the characteristics of ZFPs, we obtained an 18 bp DNA sequence 5'-cggccggctggacgcact-3' (see the sequence in the frame in Seq. 1), which was considered to be the target sequence interacting with the artificial ZFP. This target sequence was then submitted to "Design a Zinc Finger Protein". After setting 

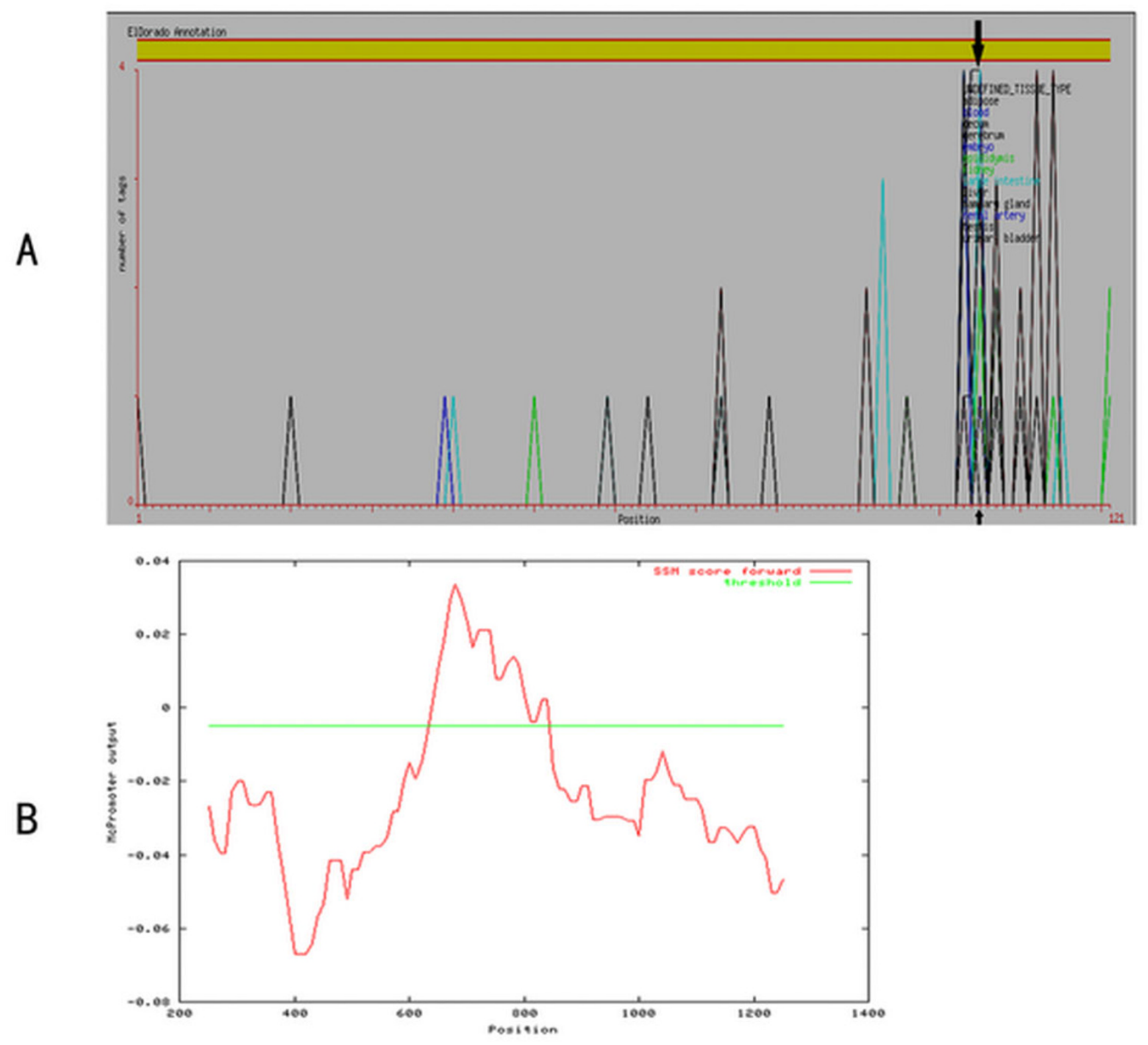

Figure I

A: The abscissa represents the region most probably containing the TSS, and the ordinate represents the score for each site. The higher the score, the more likely the site is to be the TSS. Analysis of the 121 bp sequence showed that the score of a site (located in the genomic NC_000006 138230088) reached a peak value (indicated by arrow). This site was probably the TSS of the A20 gene. B: Analysis by McPromoter based on a Markov model suggested that the presumptive promoter of the A20 gene might be located within the genomic NC_000006 I38229786 - I 38230 I82 and be 396 bp in length. The green straight line represents the threshold value. The part of the score above the threshold suggests a possible presumptive promoter.

parameters, we obtained the full-length amino acid sequence of the ZFP (see Seq. 2), which comprised 176 amino acids. In the sequence, the gray bold letters separately refer to the $-1,+3$ and +6 sites in the $\alpha$-helix of the zinc finger.

\section{LEPGEKP YKCPECGKSFSTHLDLIRHQRTH}

TGEKP YKCPECGKSFSHTGHLLEHQRTH

TGEKP YKCPECGKSFSQRAHLERHQRTH 


\section{TGEKP YKCPECGKSFSTSGELVRHQRTH}

TGEKP YKCPECGKSFSRNDTLTEHQRTH

\section{TGEKP YKCPECGKSFSRSDKLTEHQRTH}

\section{TGKKT S}

\section{Optimization of presumptive nucleotide sequence of ZFP} The presumptive nucleotide sequence (see Seq. 3) of the ZFP was optimized at Graphical Codon Usage Analyser [26].

5-ctggaaccgggtgaaa aaccttacaaatgtccgga atgtggtaaaagcttcagta cccacctggatctgatccgccaccagcgcactcaca ccggcgaaaaacgtacaagtgcc cagaatgtggcaagagtttcagccacaccggccacc tgctggaacatcagcgtacccataccggtgagaaaccgt acaaatgtcctgaatgcggcaaaagcttt agtcagcgcgcccacctggaacgccaccagcgtacccat accggcgagaaaccgtataagtgtccggaatgcggcaagag

ctttagcaccagcggcgaactggtgcgccaccagcgcacccata ccggtgaaaagccatataaatgccogaatgcggtaaaagtttcagccgcaac gatacctgaccgaacatcagcgtacccacaccggtgagaagccgtataaatgtcca gaatgtggtaagagttttagtcgcagcgataaactgaccgaacaccaacgcaccatacc ggtaaaaagaccagc-3 (528 bp)

\section{ZFP sequence characterization and structural modeling} Analysis by PepTool Lite indicated that the 176-amino acid sequence of the ZFP involves 6 regularly aligned, contiguous $\mathrm{Cys}_{2} \mathrm{His}_{2}$-type zinc fingers, which show the typical $\beta-\beta-\alpha$ secondary structure (Fig. 2A). ZFP characterization using ExPASy Proteomics tools - ProtScale suggested that the ZFP has a theoretical isoelectric point of 9.19 and a molecular weight of $19.99 \mathrm{kDa}[27,28]$. Bulkiness analysis indicated significant decreases in the scores of the dense $\alpha$-helix of each zinc finger in ZFP, and the scores of the bulky $\beta$-fold were the highest [27]. Analysis of polarity and accessible residues also confirmed that the constructed ZFP sequence possessed the structural characteristics and possible biological activity of a typical zinc finger. In the absence of $\mathrm{Zn}^{2+}$ (a stabilizer of the zinc finger structure), the instability index of ZFP was calculated as 42.46, indicating structural instability. The theoretical half-life of the ZFP was predicted to be $5.5 \mathrm{~h}$ in eukaryotic cells but only $2 \mathrm{~min}$ in $E$. coli. Hence, if the ZFP is to be expressed in prokaryotic cells, protease-deficient bacteria should be used to decrease catabolism of the heterogeneous $\mathrm{ZFP}$; moreover, $\mathrm{Zn}^{2+}$ should be added to the medium to maintain the structural stability of the ZFP.

3-D structural modeling of the ZFP backbone was carried out using the online Robetta server (Washington University) $[29,30]$. The ZFP file (in PDB format) sent via e-mail was converted by RASWIN software to JPG format. The image showed 6 contiguous zinc fingers, forming an orderly helical structure (Fig. 2B).

\section{Construction of pIRES2-EGFPIZFP-flag recombinants}

The insert DNA, a 573 bp fragment product amplified by PCR, was subcloned into the plasmid vectors pIRES2EGFP after double digestion by the restriction endonucleases XhoI and BamHI. The constructed recombinants underwent restriction digest analysis with XhoI and the

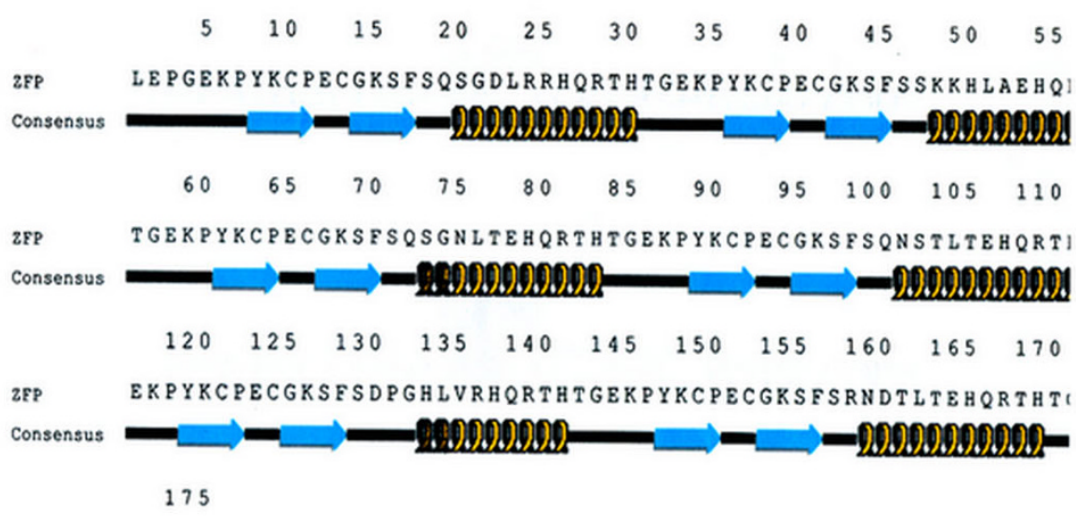

A

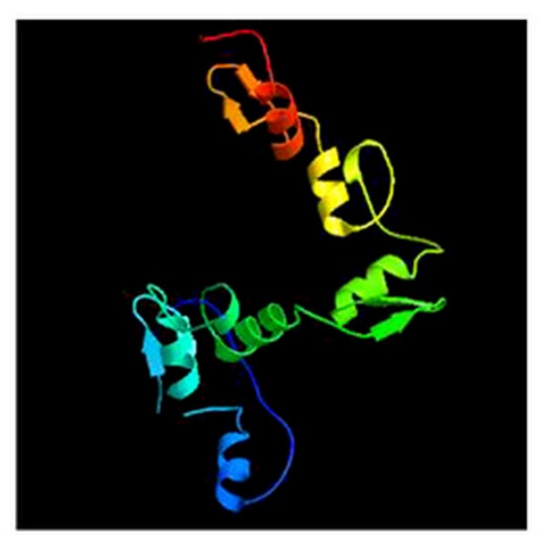

B

\section{Figure 2}

A: Analysis by PepTool Lite indicated that the I76-amino acid sequence of the ZFP involves 6 regularlyaligned, contiguous $\mathrm{Cys}_{2} \mathrm{His}_{2}$-type zinc fingers, which showed the typical $\beta-\beta-\alpha$ secondary structure.B: Illustration of 6 contiguous $\beta-\beta-\alpha$ zinc fingers constituted the 3-D structural modeling of the backbone of the ZFP, carried out on the online Robetta server. This orderly helical structure would be able to bind specially in the DNA major groove. 
digestion products were subjected to agarose gel electrophoresis; the result showed a clear electrophoretic band at approximately $5.8 \mathrm{~kb}$, consistent with the theoretical results. Colony PCR analysis and the recombinant sequencing results showed that the construction of pIRES2-EGFP/ZFP-flag was successful.

\section{Expression of green fluorescent protein by COS-7 cells} In the pIRES2-EGFP/ZFP-flag transfection group, cells emitting green fluorescence were observed under the microscope $12 \mathrm{~h}$ after transfection; $24 \mathrm{~h}$ after transfection there were significantly more of these cells, up to dozens in each visual field $(100 \times)$, mostly emitting bright green fluorescence. The fluorescence intensity reached a peak 48 $\mathrm{h}$ after transfection, and the cells were found to form masses or pairs, with no significant differences between the nuclear and cytoplasmic fluorescence intensities. In order to determine the transfection efficiency, the cells were counted in 10 random visual fields $(100 \times) 36 \mathrm{~h}$ after transfection. Of the 519 cells counted, 227 emitted green fluorescence $(43.7 \%)$. In the pIRES2-EGFP control group, green fluorescence emitting cells were first observed $8 \mathrm{~h}$ after transfection. Moreover, the number of cells emitting green fluorescence and the fluorescence intensity were higher in the pIRES2-EGFP control group than in the
pIRES2-EGFP/ZFP-flag transfection group at various times of cell culture. In the control group, no green fluorescence was observed (Fig. 3).

\section{ZFP mRNA and protein were expressed significantly in COS-7 cells after transfected}

Twenty-four hours after plasmid transfection, total RNA was prepared from Cos7 cells. Electrophoresis showed that the total RNA was not degraded. As expected, agarose gel electrophoresis indicated a marked specific band at 392 bp after RT-PCR amplification, consistent with the theoretical results (Fig. 4A). This result was not observed in the pIRES2-EGFP control group. Forty-eight hours after plasmid transfection, Western blotting showed obvious protein bands in the pIRES2-EGFP/ZFP-flag transfection group, but not in the pIRES2-EGFP control group, demonstrating successful expression of the ZFP fusion Flag (Fig. 4B).

\section{DNA binding activity}

EMSA experiments showed corresponding lag bands in the group of protein-specific probe binding reactions (lanes 1 to 3 ), but no relevant band in the negative control reaction array. In the Super-Shift reaction array, a lag band of the "antigen-antibody-specific probe complex" was

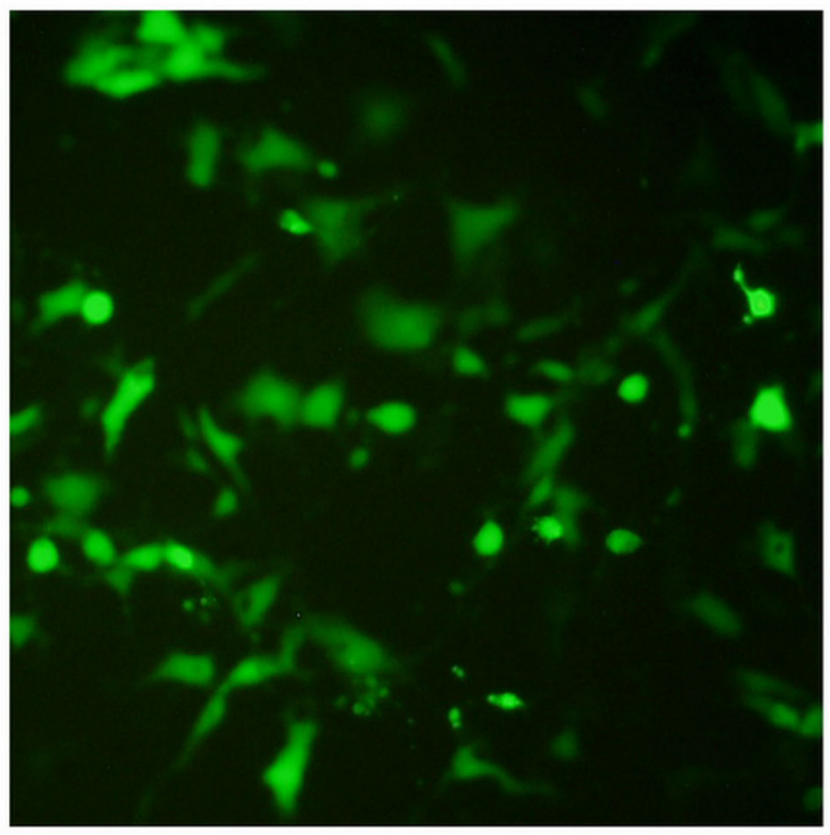

A

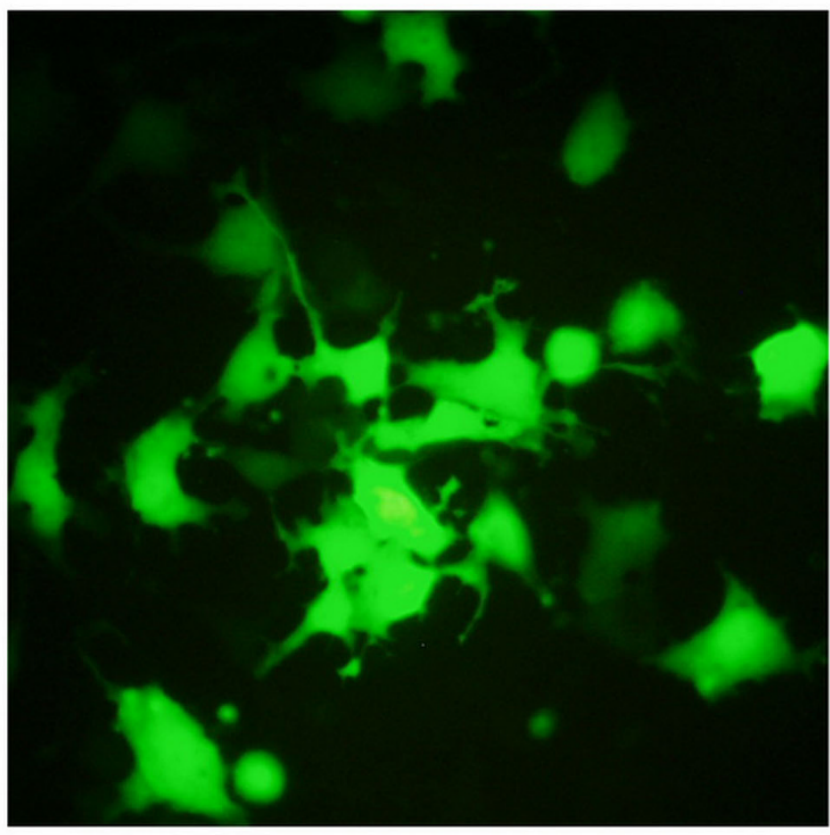

B

Figure 3

Expression of green fluorescent protein in COS-7 cells transfected with recombinant plasmids pIRES2-EGFP/ ZFP-flag seen through the fluorescence microscope. A: Expression $24 \mathrm{~h}$ after transfection (I00x). B: Expression $36 \mathrm{~h}$ after transfection (200x). The result of the pIRES2-EGFP control group was omitted. 


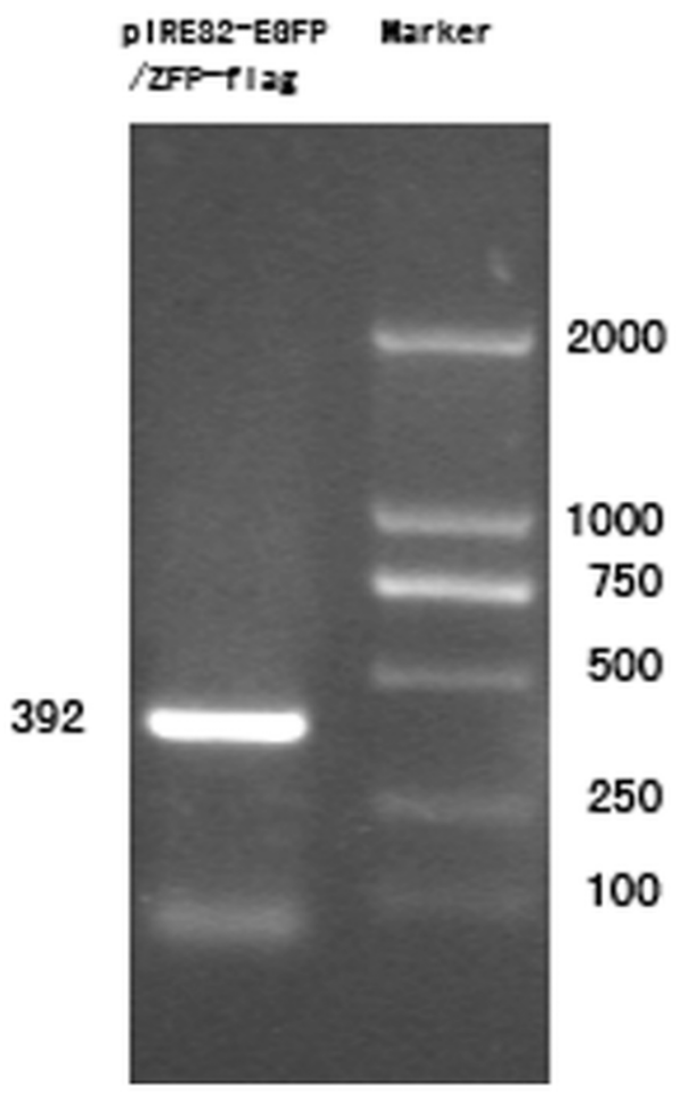

A

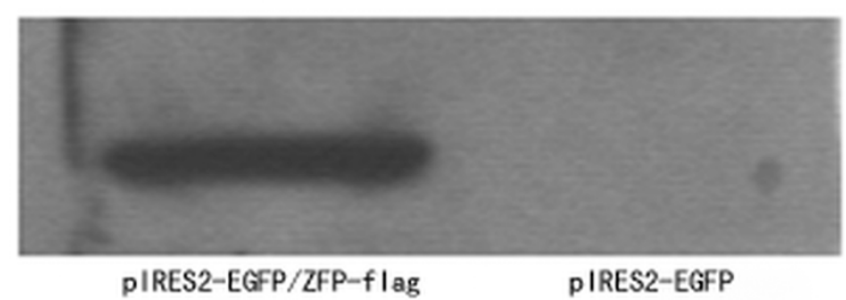

B

\section{Lane1 Lane2 Lane3 Lane4 Lane5}

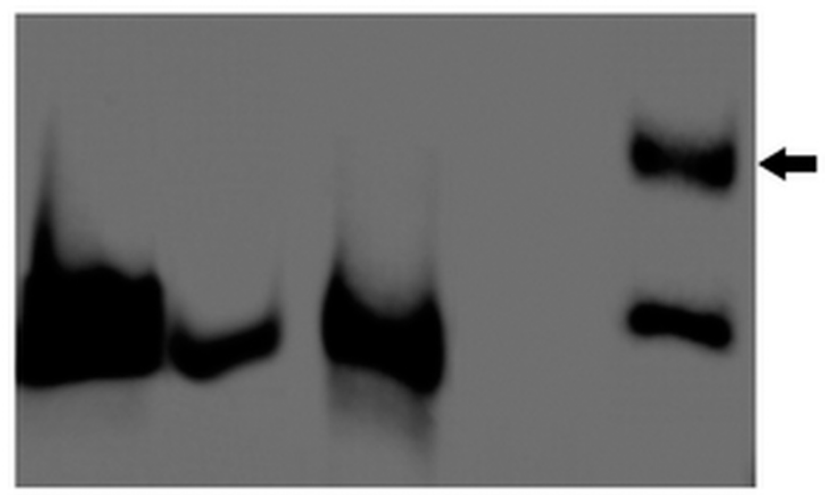

C

\begin{abstract}
Figure 4
A: RT-PCR was performed on total RNA isolated from COS7 cells transiently transfected for $24 \mathrm{~h}$ with pIRES2-EGFP/ZFP-flag. Agarose gel electrophoresis indicated an obvious specific amplification band at 392 bp. B: Western blotting. The pIRES2-EGFP/ZFP-flag transfection group showed clear protein bands $48 \mathrm{~h}$ after transfection, but these did not appear in the pIRES2-EGFP control group. C: Electrophoretic mobility shift assay. Lane I: sample reaction array. Lane 2: "cold" competition assay of probes array. Lane 3: "cold" competition assay of mutated probes array. Lane 4: negative control reaction array. Lane 5: Super-Shift reaction array. A lag band of "antigen-antibody-specific probe complex" was observed (indicated by arrow).
\end{abstract}

observed (Fig. 4C). This experiment demonstrates that the ZFP expressed by COS7 cells can bind to specific probes and possesses biological activity and, at the same time, has a correctly refolded 3-D structure. These findings provide clear evidence for the protein's ability to bind specifically to the target DNA.

\section{Discussion}

With the rapid development of bioinformatics, the volume of biological information in various databases has grown to huge proportions. Most of this information could formerly be obtained only through laboratory research. However, theoretically ideal biological information can now be obtained through efficient use of bioin- formatics tools [11]. This has allowed the research tools for modern genetics, biology, biochemistry and pharmacology to expand [31-33].

These developments open new avenues in gene therapy by designing proteins that do not exist naturally but can regulate specific genes by simulating the structure of a naturally-occurring transcription factor. Gene expression is regulated at the transcriptional level primarily (and most economically and effectively) by transcription factors that bind to DNA [3]. To intervene in gene expression, the promoter of the gene of interest must first be studied. A gene promoter contains a series of NA sequence elements adjoining the transcriptional start site (TSS), which 
directly activate or inhibit transcription. Hence, the position of a promoter can be located through the TSS, once this has been identified. Therefore, precise location of the TSS is the primary requirement for analyzing promoters and transcriptional regulation. The promoter of a gene is frequently located within $100 \mathrm{bp}-300 \mathrm{bp}$ upstream of the TSS, and a typical core promoter includes a DNA sequence covering about $-40 \mathrm{bp}-+50 \mathrm{bp}$ of the TSS [34]. We defined the A20 gene promoter and its TSS theoretically through bioinformatics analysis and showed many potentially important regulatory elements in the A20 promoter region.

An ATF consists of a DNA-binding domain and a functional domain [4-6]. The most crucial step in constructing an effective ATF is to design the DNA-binding domain successfully. Since Miller et al. found that the transcription factor TFIIIA was a $\mathrm{Cys}_{2} \mathrm{His}_{2}$-type zinc finger protein in Xenopus oocytes[35]; hundreds of proteins controlling gene replication and transcription have been found containing zinc finger motif. The Barbas Laboratory developed a series of artificial zinc finger domains by the phage display technique, which recognized different members of the 5'-NNN-3' family of DNA sequences (5'-GNN-3', 5'CNN-3', 5'-ANN-3', 5'-TNN-3') [9-11,36,37]. They also used them to construct different ATFs. Thus, the Zinc Finger Tools identify individual zinc fingers as a module of the protein's structural domain, and each of the contiguous DNA triplets can specifically recognize an independent zinc finger module [12]. On this basis, as multiple zinc finger modules are integrated into a segment of polypeptide or protein sequence, the resulting sequence can in theory bind a DNA sequence selectively. ZF Tools greatly lower the workload of researchers studying ZFPs. Since the working data of ZF Tools are the results of phage display library screening, the results obtained are theoretically credible; nevertheless, they have to be verified in the laboratory.

Structural modeling of proteins by homologous sequence comparison and alignment is relatively reliable. This method is based on the principle that the 3-D structure of a protein is more conserved than the primary structure[29]. Given this viewpoint, it is essential to acquire the crystal structures of homologous proteins as template. In fact, because of the increasing number of structural determinations of ZFPs, homologous modeling of new proteins containing zinc finger domains may be carried out. We successfully obtained the PDB files of ZFPs from the 3D-JIGSAW, Swiss-Model and Robetta online servers.

\section{Conclusion}

It is feasible to design specific zinc finger proteins by bioinformatics methods. This study provides a basis for arti- ficially designing further complete transcription factors and intervening in the expression of the A20 gene.

\section{Methods \\ DNA synthesis}

All the primers and the ZFP gene sequence subcloned in the pUC19 plasmid were synthesized by Shanghai Sangon Biological Engineering Technology \& Services Co., Ltd.

\section{Enzymes and reagents}

PCR product purification kits were purchased from Beijing Biodev-Tech, gel recovery kits and plasmid extraction kits from Invitrogen, RT-PCR kits from Hangzhou Boer, and nuclear and cytoplasmic protein extraction kits from Beyotime. RPMI-1640 medium was purchased from Gibco, fetal bovine serum from Hyclone, DOTAP liposomes from Roche, Trizol reagent from Invitrogen, antiFlag antibody from the Antibody Research Center of Shanghai Institutes for Biological Sciences, and HRPlabeled secondary antibody from Santa Cruz. Taq DNA polymerase and dNTPs were purchased from Promega, restriction endonucleases, T4 DNA ligase and T4 polynucleotide kinase from Takara, prestained standard protein markers from $\mathrm{MBI}, \mathrm{ZnCl}_{2}$, and nitrocellulose (NC) membrane and X-ray film from Sigma.

\section{Analysis of A20 gene promoter region}

The A20 gene (GeneID:7128) and related sequences were retrieved from the National Center for Biotechnology Information[38]. The A20 gene promoter was analyzed using the sequence processing tools TFSEARCH, TESS, Gene2Promoter and McPromoter.

\section{Design of presumptive nucleotide sequence of ZFP}

We logged on to the Zinc Finger Tools server of the Barbas Laboratory of the Scripps Research Institute, submitted A20 promoter-related sequences, and set up parameters to obtain the specific target site of the A20 gene promoter region and the amino acid sequence of a ZFP. This amino acid sequence was then reverse-translated into a nucleotide sequence, and all codons in that sequence were optimized. The full-length optimized sequence was sent to Shanghai Sangon Company for full-gene synthesis.

\section{ZFP sequence characterization and structural modeling}

The characterization and structural analysis of the ZFP were carried out using PepTool Lite 1.1 and ProtScale. Homologous modeling of the ZFP tertiary structure was conducted using Robetta.

\section{Construction of pIRES2-EGFPIZFP-flag eukaryotic expression plasmids}

ZFP gene fragments were amplified by PCR, using pUC19ZFP as a template, and the primers were designed as follows. Forward: 5'-CCGCTCGAGATGGACTACAAGGAC- 
GACGATGACAAGCTGGAACCGGGTGA-3'; reverse: 5'CGGGGATCCGCTGGTCTTTTTACCGGTATGC-3'

(framed: restriction sites; underlined: Flag tag-encoding sequence). PCR products and pIRES2-EGFP plasmids were doubly-digested with the restriction endonucleases XhoI and BamHI. According to the design protocol, $\mathrm{T}_{4}$ DNA ligase was used to ligate the target fragments, and the ligated products were used to transform competent TOP10 E. coli. The recombinants were preliminarily identified by restriction digest analysis and colony PCR (PCR primers, forward: 5'-CCCAGAATGTGGCAAGAGTT-3', reverse: 5'-TGCGTTGGTGTTCCGTCAGT-3'). The bacterial suspensions containing positive recombinants were sent to Shanghai Sangon Company for sequencing. The recombinants identified were named pIRES2-EGFP/ZFPflag.

\section{Transfection of COS-7 cells}

COS-7 cells were routinely cultured in 24-well plates in RPMI-1640 medium supplemented with 7\% fetal bovine serum, $0.1 \mathrm{mM}$ (final concentration) $\mathrm{ZnCl}_{2}$ at $37^{\circ} \mathrm{C}$ and $6 \% \mathrm{CO}_{2}$ in a humidified atmosphere. At $60 \%$ cell confluence, plasmid transfection was carried out according to the instructions on the DOTAP kit. In the experimental group, COS-7 cells were transfected with the recombinant pIRES2-EGFP/ZFP-flag plasmids, and in the positive control group the cells were transfected with void pIRES2EGFP plasmids. In the blank group, liposomes instead of plasmids were added.

\section{Analysis of ZFP mRNA expression using RT-PCR}

Cos7 cells were transfected for $24 \mathrm{~h}$ with the recombinant pIRES2-EGFP/ZFP-flag plasmids. Total RNA was isolated with Trizol Reagent (Invitrogen) according to the manufacturer's instructions. RNA purity was assessed by electrophoresis on formaldehyde-denaturing agarose gels and measuring the $\mathrm{OD}_{260} / \mathrm{OD}_{280}$ absorption ratio. Five micrograms of total RNA was used to synthesize the first strand cDNA under the conditions recommended by the manufacturer. Ten percent of the first strand CDNA was used as template for the PCR. The primers used for RT-PCR amplification were the same as those used for colony PCR. The amplified fragment should be 392 bp in length. PCR amplification products were identified by $1.5 \%$ agarose gel electrophoresis.

\section{Analysis of ZFP protein expression using western blotting assay}

Forty-eight hours after plasmid transfection, the cells were collected and lyzed to extract total cellular protein. The processed protein samples were subjected to SDS-polyacrylamide gel electrophoresis and transferred on to a nitrocellulose membrane. The membrane was incubated overnight with anti-Flag monoclonal antibody at $4^{\circ} \mathrm{C}$ and with horseradish peroxidase (HRP)-labeled goat anti- mouse secondary antibody at room temperature for $2 \mathrm{~h}$. Cells transfected with void pIRES2-EGFP plasmids were used as control.

\section{Electrophoretic mobility shift assay (EMSA)}

On the basis of the target sequence selected from the A20 gene promoter region, a pair of specific probes was designed as follows: ATCCCGGCCGGCTGGACGCACTTACA, and a pair of mutated probes was also designed as follows: ATCCTGGCCGGCTAGACGCACTTACA. The specific probes were labeled with $[\gamma-32 \mathrm{P}]$-ATP $(3,000 \mathrm{Ci} / \mathrm{mmol}$ at $10 \mathrm{mCi} / \mathrm{ml}$ ) and purified. Forty-eight hours after plasmid transfection, cell protein was extracted. Electrophoretic mobility shift assays were conducted in accordance with the protocol on the kit.

\section{Abbreviations}

ATF, Artificial transcription factors; TNFAIP3, Tumor Necrosis Factor, alpha-Induced Protein 3; TNF, Tumor necrosis factor; NFкB, Nuclear factor kappa-B; ZF, Zinc finger; ZFP, Zinc finger protein; AZP, artificial zinc finger protein; TSS, Transcription start site; EGFP, Enhanced Green Fluorescent Protein; HRP, Horseradish peroxidase; EMSA, Electrophoretic mobility shift assay.

\section{Authors' contributions}

YW analyzed the structure and the sequence composition of the A20 gene, designed the ZFP structural domain by bioinformatic means, and YW performed the construction of pIRES2-EGFP/ZFP-flag recombinant. CHZ conceived the study, and helped in the writing of the manuscript. CLH and XPC performed the COS7 cell culture and transfection, including Rt-PCR, Western blot, with the help of YW. DJY gave advice in the proceeding of the experiment and edited the manuscript. All authors read and approved the final manuscript.

\section{Acknowledgements}

We thank Dr. Jun-lei Zhang for her gift of COS-7 cells and Dr. Gang Huang for his gift of pIRES2-EGFP plasmids (both of them work at the Third Military Medical University). This work was supported by a grant from the National Natural Science Foundation of China (No. 30472027, 30770575) and $\mathrm{Hi}$-Tech Research and Development Program of China (863

No:2006AA03Z438).

\section{References}

I. Beerli RR, Dreier B, Barbas CF 3rd: Positive and negative regulation of endogenous genes by designed transcription factors. Proc Natl Acad Sci USA 2000, 97: 1495-500.

2. Segal DJ, Dreier B, Barbas CF 3rd: Toward controlling gene expression at will: specific regulation of the erbB-2/HER-2 promoter by using polydactyl zinc finger proteins constructed from modular building blocks. Proc Natl Acad Sci USA 1998, 95: |4628-33.

3. Zhao $\mathrm{XH}$, Zhu XD, Huang PT: Artificial transcription factors as tools for gene expression manipulation. Sheng Wu Gong Cheng Xue Bao 2005, 21:34I.

4. Beerli RR, Barbas CF 3rd: Engineering polydactyl zinc-finger transcription factors. Nat Biotechnol 2002, 20:|35-| 4 I. 
5. Quon DV, Delgadillo MG, Khachi A, Smale ST, Johnson PJ: Similarity between a ubiquitous promoter element in an ancient eukaryote and mammalian initiator elements. Proc Natl Acad Sci USA 1994, $91: 4579-4583$.

6. Shieh JC, Cheng YC, Su MC, Moore M, Choo Y, Klug A: Tailormade zinc-finger transcription factors activate FLOII gene expression with phenotypic consequences in the yeast Saccharomyces cerevisiae. PLOS ONE 2007, 2(I):e746.

7. Lee DK, Seol W, Kim JS: Custom DNA-Binding Proteins and Artificial Transcription Factors. Current Topics in Medicinal Chemistry 2003, 3:339-353.

8. Falke D, Fisher M, Ye D, Juliano RL: Design of artificial transcription factors to selectively regulate the pro-apoptotic bax gene. Nucleic Acids Research 2003, 3 I (3):e IO.

9. Segal DJ, Dreier B, Beerli RR, Barbas CF 3rd: Toward controlling gene expression at will: Selection and design of zinc finger domains recognizing each of the 5'-GNN-3' DNA target sequences. Proc Natl Acad Sci USA 1999, 96:2758-2763.

10. Dreier B, Fuller RP, Segal DJ, Lund CV, Blancafort P, Huber A, Koksch $B$, Barbas CF 3rd: Development of zinc finger domains for recognition of the 5'-CNN-3' family DNA sequences and their use in the construction of artificial transcription factors. J Biol Chem 2005, 280:35588-35597.

II. Dreier B, Beerli RR, Segal DJ, Flippin JD, Barbas CF 3rd: Development of zinc finger domains for recognition of the 5 '-ANN-3' family of DNA sequences and their use in the construction of artificial transcription factors. I Biol Chem 200I, 276:29466-29478.

12. Mandell JG, Barbas CF 3rd: Zinc Finger Tools: custom DNAbinding domains for transcription factors and nucleases. Nucleic Acids Res. (Web Server issue) 2006, 34:W516-523.

13. Beerli RR, Schopfer U, Dreier B, Barbas CF 3rd: Chemically regulated zinc finger transcription factors. J Biol Chem 2000, 275:32617-32627.

14. Dixit VM, Green S, Sarma V, Holzman LB, Wolf FW, O'Rourke K, Ward PA, Prochownik EV, Marks RM: Tumor necrosis factoralpha induction of novel gene products in human endothelial cells including a macrophage-specific chemotaxin. J Biol Chem 1990, 265:2973-2978.

15. Lee EG, Boone DL, Chai S, Libby SL, Chien M, Lodolce JP, Ma A: Failure to regulate TNF-induced NF-kappa-B and cell death responses in A20-deficient mice. Science 2000, 289:2350-2354.

16. Zhang B, Guan CC, Chen WT, Zhang P, Yan M, Shi JH, Qin CL, Yang Q: A20 inhibits human salivary adenoid cystic carcinoma cells invasion via blocking nuclear factor-kappaB activation. Chin Med J (Engl) 2007, I 20:1830-1835.

17. Cooper JT, Stroka DM, Brostjan C, Palmetshofer A, Bach FH, Ferran C: A20 Expression Inhibits Endothelial Cell Activation. Transplantation Proceedings 1997, 29:88I.

18. Grey ST, Arvelo MB, Hasenkamp WM, Bach FH, Ferran C: Adenovirus-mediated gene transfer of the anti-apoptotic protein A20 in rodent islets inhibits IL-I beta-induced NO release. Transplantation Proceedings 1999, 3 1:789.

19. Klinkenberg M, Van Huffel S, Heyninck K, Beyaert R: Functional redundancy of the zinc fingers of $A 20$ for inhibition of NF- $K B$ activation and protein-protein interactions. FEBS Letters $200 \mathrm{I}$ 498:93-97.

20. TFSEARCH [http://www.cbrc.jp/research/db/TFSEARCH.html]

21. TESS [http://www.cbil.upenn.edu/cgi-bin/tess/tess?RQ=WEL COME]

22. Gene2Promoter [http://www.genomatix.de/index.html]

23. Krikos A, Laherty CD, Dixit VM: Transcriptional activation of the tumor necrosis factor alpha-inducible zinc finger protein, A20, is mediated by kappa B elements. I Biol Chem 1992, 267: $1797 \mid-17976$

24. McPromoter [http://tools.genome.duke.edu/generegulation/ McPromoter/]

25. ZF Tools [http://www.scripps.edu/mb/barbas/zfdesign/zfdesign home.php]

26. Graphical Codon Usage Analyser [http://gcua.schoedl.de/]

27. ProtScale [http://www.expasy.ch/tools/]

28. Wilkins MR, Gasteiger E, Bairoch A, Sanchez JC, Williams KL, Appel RD, Hochstrasser DF: Protein identification and analysis tools in the ExPASy server. Methods Mol Biol 1999, I | 2:53 |-52.

29. Robetta [http://robetta.bakerlab.org/]
30. Kuhlman B, Baker D: Native protein sequences are close to optimal for their structures. Proc Natl Acad Sci USA 2000, 97:10383-10388.

31. Eberhardy SR, Goncalves J, Coelho S, Segal DJ, Berkhout B, Barbas CF $3 \mathrm{rd}$ : Inhibition of human immunodeficiency virus type I replication with artificial transcription factors targeting the highly conserved primer-binding site. I Virology 2006, 80:2873-2883.

32. Bae KH, Kwon YD, Shin HC, Hwang MS, Ryu EH, Park KS, Yang HY, Lee DK, Lee Y, Park J, Kwon HS, Kim HW, Yeh BI, Lee HW, Sohn $\mathrm{SH}$, Yoon J, Seol W, Kim JS: Human zinc fingers as building blocks in the construction of artificial transcription factors. Naure Biotechnology 2003, 2 I(3):275-280.

33. Lee DK, Park JW, Kim YJ, Kim J, Lee Y, Kim J, Kim JS: Toward a functional annotation of the human genome using artificial transcription factors. Genome Research 2003, 13:2708-27I6.

34. Javahery R, Khachi A, Lo K, Zenzie-Gregory B, Smale ST: DNA sequence requirements for transcriptional initiator activity in mammalian cells. Mol Cell Biol 1994, 14:1 I6-127.

35. Miller J, Mclachlan AD, Klug A: Repetitive zinc-binding domains in the protein transcription factor IIIA from Xenopus oocytes. J EMBO 1985, 4(6):1609-1614.

36. Dreier B, Segal DJ, Barbas CF 3rd: Insights into the molecular recognition of the 5'-GNN-3' family of DNA sequences by zinc finger domains. J Mol Biol 2000, 303:489-502.

37. Blancafort P, Magnenat L, Barbas CF 3rd: Scanning the human genome with combinatorial transcription factor libraries. Nat Biotechnol 2003, 21:269-74.

38. NCBI [http://www.ncbi.nlm.nih.gov]
Publish with Biomed Central and every scientist can read your work free of charge

"BioMed Central will be the most significant development for disseminating the results of biomedical research in our lifetime. "

Sir Paul Nurse, Cancer Research UK

Your research papers will be:

- available free of charge to the entire biomedical community

- peer reviewed and published immediately upon acceptance

- cited in PubMed and archived on PubMed Central

- yours - you keep the copyright
BiolMedcentral 